\title{
COSMOLOGICAL FRAMES FOR THEORIES WITH ABSOLUTE PARALLELISM
}

\author{
FRANCO FIORINI \\ Instituto de Astronomía y Física del Espacio, C.C. 67, Suc. 28, 1428 Buenos Aires, Argentina \\ franco@iafe.uba.ar \\ RAFAEL FERRARO* \\ Instituto de Astronomía y Física del Espacio, C.C. 67, Suc. 28, 1428 Buenos Aires, Argentina \\ and \\ Departamento de Física, Facultad de Ciencias Exactas y Naturales, \\ Universidad de Buenos Aires, Ciudad Universitaria, Pabellón I, 1428 Buenos Aires, Argentina \\ ferraro@iafe.uba.ar
}

Received 29 May 2011

\begin{abstract}
The vierbein (tetrad) fields for closed and open Friedmann-Robertson-Walker cosmologies are hard to work out in most of the theories featuring absolute parallelism. The difficulty is traced in the fact that these theories are not invariant under local Lorentz transformations of the vierbein. We illustrate this issue in the framework of $f(T)$ theories and Born-Infeld determinantal gravity. In particular, we show that the early Universe as described by the Born-Infeld scheme is singularity free and naturally inflationary as a consequence of the very nature of Born-Infeld gravitational action.
\end{abstract}

Keywords: Born-Infeld; teleparallelism; gravity.

PACS numbers: 11.25.Hf, 123.1K

\section{Introduction}

In the last decade a wide variety of modified theories of gravity has been studied with the aim of solving or smoothing some puzzling features of conventional gravity and cosmology. The reasons for considering such a modified gravitational schemes rely mainly on two damages that are intrinsic features of general relativity (GR). On one hand, it is widely accepted by high energy physicists that the description of the gravitational field provided by general relativity must be doomed at scales of the order of the Planck length, where the spacetime structure itself must be represented in terms of a quantum regime. On the other hand, and in the opposite extreme of the physical phenomena, GR also faces an intriguing dilemma in connection with the late cosmic speed up stage of the Universe.

* Member of Carrera del Investigador Científico of CONICET 
One of the newest extended theories of gravity is the so called $f(T)$ gravity, which is a theory formulated in a spacetime possessing absolute parallelism. ${ }^{1,2}$ The first investigations within this framework can be traced back to Refs. 3-5, where an ultraviolet deformation of Einstein gravity was considered with the aim of smoothing singularities, namely the initial singularity of Friedmann-Robertson-Walker (FRW) cosmological models. After that, the attention was focused in low energy deformations of GR which serve to tackle those aspects of the cosmological evolution connected with the late speed up stage of the universe in spatially flat models. ${ }^{6-22}$ Quite more recently, some fundamental aspects of $f(T)$ theories, like the presence of extra degrees of freedom and the lack of Lorentz invariance, had been addressed in Refs. 23-27. In spite of their success in smoothing the Big-Bang singularity, ${ }^{3}$ the ultraviolet deformations in the context of the $f(T)$ theories fail to smooth singularities of GR vacuum solutions like black holes. ${ }^{4}$ For this reason, we introduced in Ref. 28 a more general theory in which the singularities of Einstein theory receive a more appropriate treatment, even those that emerge from vacuum solutions of Einstein equations. In this framework the gravitational action is chosen, in close analogy with the Born-Infeld (BI) procedure used in electrodynamics, as the determinant of a particular combination of the torsion tensor that assures the correct low energy limit given by GR. Both the BI theory and the $f(T)$ 's mentioned above are based on actions built with just first derivatives of the dynamical field - the vierbein $e^{a}-$, so assuring second order motion equations.

Most of the gravitational theories with absolute parallelism lack the invariance under local Lorentz transformations of the vierbein. This means that they should be regarded as theories describing the dynamics of the vierbein field rather than the metric field (which is invariant under local Lorentz transformations of the vierbein). As a consequence, the symmetry of the metric tensor is not enough to suggest the form of the vierbein, which could be elusive in many physically meaningful solutions. In this article we will examine this issue by working out the proper vierbein field for closed and open FRW cosmologies. For this purpose, in Sec. 2 we introduce the teleparallel equivalent of general relativity, that constitutes the geometrical pillar on which the $f(T)$ and BI theories of gravity, also summarized there, are built. In Sec. 3 we explain the lack of invariance of these theories under local Lorentz transformation of the vierbein field in a cosmological context, discuss about the meaning of this feature, and work out the proper vierbein field for closed and open FRW cosmologies. Finally, in Sec. 4 we display the conclusions.

\section{Gravitational Theories with Absolute Parallelism: Two Examples}

In this section we will focus the attention on two gravitational schemes based on absolute parallelism. On one hand, we will discuss a family of modified teleparallel theories constructed as a deformation of the Einstein-Hilbert Lagrangian in Weitzenböck spacetime - the so called $f(T)$ gravities. On the other hand, we will 
consider a quite different ultraviolet modification of GR with determinantal structure: Born-Infeld gravity. We will start by introducing the geometrical concepts that serve as the building blocks of both schemes.

The basic idea is that gravity can be described by providing the spacetime with a torsion $T^{a}=d e^{a}, a=0, \ldots, 3$, where $\left\{e^{a}\right\}$ is a vierbein (a basis of the cotangent space) in a 4 -dimensional manifold. The vierbein $\left\{e^{a}\right\}$ is the co-frame of an associated basis $\left\{e_{a}\right\}$ in the tangent space. If $e_{\mu}^{a}$ and $e_{a}^{\mu}$ are respectively the components of the 1 -forms $e^{a}$ and the vectors $e_{a}$ in a given coordinate basis, then the relation between frame and co-frame is expressed as

$$
e_{\mu}^{a} e_{b}^{\mu}=\delta_{b}^{a} .
$$

Contracting with $e_{a}^{\nu}$ one also gets

$$
e_{a}^{\nu} e_{\mu}^{a}=\delta_{\mu}^{\nu} .
$$

The components $T^{\lambda}{ }_{\mu \nu}$ of the torsion tensor in the coordinate basis is related to the 2-forms $T^{a}$ through the equation

$$
T_{\mu \nu}^{\lambda} \equiv e_{a}^{\lambda} T_{\mu \nu}^{a}=e_{a}^{\lambda}\left(\partial_{\nu} e_{\mu}^{a}-\partial_{\mu} e_{\nu}^{a}\right)
$$

This means that the spacetime is endowed with a connection

$$
\Gamma_{\mu \nu}^{\lambda}=e_{a}^{\lambda} \partial_{\nu} e_{\mu}^{a}+\text { terms symmetric in } \mu \nu,
$$

(since $T_{\mu \nu}^{\lambda} \equiv \Gamma_{\nu \mu}^{\lambda}-\Gamma_{\mu \nu}^{\lambda}$ ). The first term in Eq. (4) is the Weitzenböck connection. The metric is introduced as a subsidiary field given by

$$
g_{\mu \nu}(x)=\eta_{a b} e_{\mu}^{a}(x) e_{\nu}^{b}(x),
$$

where $\eta_{a b}=\operatorname{diag}(1,-1,-1,-1)$. Eq. (5) can be inverted with the help of Eq. (1) to obtain

$$
\eta_{a b}=g_{\mu \nu}(x) e_{a}^{\mu}(x) e_{b}^{\nu}(x),
$$

which means that the vierbein is orthonormal.

Teleparallelism uses the Weitzenböck spacetime, where the connection is chosen as

$$
\Gamma_{\mu \nu}^{\lambda}=e_{a}^{\lambda} \partial_{\nu} e_{\mu}^{a}
$$

As a consequence of the choice of the Weitzenböck connection (7), the Riemann tensor is identically null. So the spacetime is flat: the gravitational degrees of freedom are completely encoded in the torsion $T^{a}=d e^{a}$.

In terms of parallelism, the choice of the Weitzenböck connection has a simple meaning. In fact, the covariant derivative of a vector yields

$$
\nabla_{\nu} V^{\lambda}=\partial_{\nu} V^{\lambda}+\Gamma_{\mu \nu}^{\lambda} V^{\mu}=e_{a}^{\lambda} \partial_{\nu}\left(e_{\mu}^{a} V^{\mu}\right) \equiv e_{a}^{\lambda} \partial_{\nu} V^{a}
$$

In particular, Eq. (6) implies that $\nabla_{\nu} e_{b}^{\lambda}=0$; so, the Weitzenböck connection is metric compatible. In general, Eq. (8) means that a given vector is parallel transported along a curve if its projections on the co-frame remain constant. So, the vierbein parallelizes the spacetime. Of course, this nice criterion of parallelism would 
be destroyed if local Lorentz transformations of the co-frame were allowed in the theory.

Teleparallelism is a dynamical theory for the vierbein, which is built from the torsion. According to Eq. (5), a set of dynamical equations for the vierbein also implies a dynamics for the metric. This dynamics coincides with Einstein's dynamics for the metric when the teleparallel Lagrangian density is chosen as ${ }^{29,30}$

$$
\mathcal{L}_{\mathbf{T}}\left[e^{a}\right]=\frac{1}{16 \pi G} e(T-2 \Lambda)
$$

where $e \equiv\left|e_{\mu}^{a}\right|=\sqrt{\left|g_{\mu \nu}\right|}$, and || stands for the absolute value of the determinant. In Eq. (9) we have defined the Weitzenböck invariant as

$$
T=S_{\mu \nu}^{\rho} T_{\rho}^{\mu \nu} .
$$

The tensor $S_{\mu \nu}^{\rho}$ appearing in the last equation is defined according to

$$
S_{\mu \nu}^{\rho}=\frac{1}{4}\left(T_{\mu \nu}^{\rho}-T_{\mu \nu}{ }^{\rho}+T_{\nu \mu}{ }^{\rho}\right)+\frac{1}{2} \delta_{\mu}^{\rho} T_{\sigma \nu}{ }^{\sigma}-\frac{1}{2} \delta_{\nu}^{\rho} T_{\sigma \mu}{ }^{\sigma} .
$$

In fact, the Lagrangian (9) just differs from the Einstein-Hilbert Lagrangian with cosmological constant $\mathcal{L}_{\mathbf{G R}}=-(16 \pi G)^{-1} e(R+2 \Lambda)$ in a total derivative

$$
-e R\left[e^{a}\right]=e T-2 \partial_{\nu}\left(e T_{\sigma}{ }^{\sigma \nu}\right),
$$

where $R$ is the scalar curvature for the Levi-Civita connection. When GR dresses this costume, the gravitational degrees of freedom are gathered in the torsion instead of the Levi-Civita curvature. It is a very curious and fortunate fact that both pictures enable to construct a gravitational action with the same physical content. However, it is remarkable that the Lagrangian (9) involves just first derivatives of its dynamical field, the vierbein. In some sense, the teleparallel Lagrangian picks up the essential dynamical content of Einstein theory without the annoying second order derivatives appearing in the last term of Eq. (12). Such Lagrangian is a better starting point for considering modified gravity theories, since any deformation of its dependence on $T$ will always lead to second order dynamical equations. On the contrary, the so called $f(R)$ theories lead to fourth order equations.

Analogously to the $f(R)$ scheme, a $f(T)$ theory replaces the Weitzenböck invariant $T$ in Eq. (9) with a general function $f(T)$. So, the dynamics is described by the action

$$
\mathcal{I}=\frac{1}{16 \pi G} \int d^{4} x \operatorname{e} f(T)+\int d^{4} x \mathcal{L}_{\mathcal{M}},
$$

where $\mathcal{L}_{\mathcal{M}}$ is the matter Lagrangian density. Undoubtedly, the whole family of actions gathered in (13) constitutes a vast territory worth to be explored, specially when one is aware that the dynamical equations arising by varying the action (13) with respect to the vierbein components $e_{\mu}^{a}(x)$ are of second order. This distinctive feature makes Weitzenböck spacetime a privileged geometric structure to formulate modified theories of gravitation. In fact, the dynamical equations for the vierbein tell how the matter distribution organizes the orientation of the basis $e^{a}$ at each point, 
in such a way that the field lines of $e^{a}(x)$ realize the parallelization of the manifold. After this vierbein field is obtained, one uses the assumption of orthonormality to get the metric (5).

Let us deal now with Born-Infeld gravity. This high energy modification of Einstein gravity is based on the Born-Infeld procedure used first in the context of electrodynamics, and is governed by the $n$-dimensional action in Weitzenböck spacetime given $\mathrm{by}^{28}$

$$
\mathcal{I}_{\mathrm{BIG}}=\frac{\lambda /(A+B)}{16 \pi G} \int d^{n} x\left[\sqrt{\left|g_{\mu \nu}+2 \lambda^{-1} \mathcal{F}_{\mu \nu}\right|}-\alpha \sqrt{\left|g_{\mu \nu}\right|}\right],
$$

where $\mathcal{F}_{\mu \nu}$ is quadratic in the Weitzenböck torsion, and reads

$$
\mathcal{F}_{\mu \nu}=A S_{\mu \lambda \rho} T_{\nu}^{\lambda \rho}+B S_{\lambda \mu \rho} T_{\nu}^{\lambda}{ }^{\rho},
$$

being $A$ and $B$ non-dimensional constants. Such a combination ensures the correct GR limit since both terms in $\mathcal{F}_{\mu \nu}$ have trace proportional to the Weitzenböck invariant $T=S^{\rho}{ }_{\mu \nu} T_{\rho}{ }^{\mu \nu}$. In order to show this fact, we can factor out $\sqrt{\left|g_{\mu \nu}\right|}$ from expression (14) and use the expansion of the determinant,

$$
\sqrt{\left|\mathbb{I}+2 \lambda^{-1} \mathbb{F}\right|}=1+\lambda^{-1} \operatorname{Tr}(\mathbb{F})+\mathcal{O}\left(\lambda^{-2}\right),
$$

where $\mathbb{F} \equiv \mathcal{F}_{\mu}{ }^{\nu}$ and $\mathbb{I}$ is the identity. This last equation expresses the fact that in any number of dimensions we have

$$
\left.\mathcal{I}_{\mathbf{B I G}}\right|_{\lambda \rightarrow \infty}=\frac{1}{16 \pi G} \int d^{n} x e(T-2 \Lambda) .
$$

Hence, at the lowest order we retrieve the low energy regime described by the Einstein theory with cosmological constant $\Lambda=\lambda(\alpha-1) /[2(A+B)]$.

\section{Nontrivial Frames for Spatially Curved FRW Universes}

Due to the fact that general relativity is a theory for the metric, it is invariant under local Lorentz transformations of the vierbein. However, the equivalence between Teleparallelism (9) and GR dynamics, expressed in Eq. (12), implies that $T$ changes by a boundary term under local Lorentz transformations. Because of this reason, the teleparallel equivalent of GR does not provide the manifold with a parallelization but only with a metric. On the contrary, in a $f(T)$ theory the "boundary term" in $T$ will remain encapsulated inside the function $f$. This means that a $f(T)$ theory is not invariant under local Lorentz transformations of the vierbein. So, a $f(T)$ theory will determinate the vierbein field almost completely (up to global Lorentz transformations). In other words, a $f(T)$ theory will describe more degrees of freedom than the teleparallel equivalent of GR. This is an important issue in the search for solutions to the $f(T)$ dynamical equations, since pairs of vierbein fields connected by local Lorentz transformations (i.e., leading to the same metric tensor) are inequivalent from the point of view of the theory. Clearly, the same considerations are also valid for Born-Infeld gravity because the determinantal action (14) is constructed with 
quadratic combinations of the torsion tensor, which fails to be invariant under a local Lorentz change of the tetrad.

Due to the lack of local Lorentz invariance, the vierbein fields solving the equations of theories with absolute parallelism are harder to obtain than the metric field. In fact, the considerations of symmetry about the form of the metric can be of little help to determine which among a set of locally Lorentz related frames is the right one to solve the dynamical equations. As an example, we will find here the adequate frames in order to deal with FRW Universes with non flat spatial sections. For these geometries, the line element can be described in hyper-spherical coordinates as

$$
d s^{2}=d t^{2}-\kappa^{2} a^{2}(t)\left[d(\kappa \psi)^{2}+\sin ^{2}(\kappa \psi)\left(d \theta^{2}+\sin ^{2} \theta d \phi^{2}\right)\right],
$$

where $\kappa=1$ for the closed Universe and $\kappa=i$ for the open Universe. Here, one is tempted to think that the vierbein that solves the dynamical equations could have the form

$$
\begin{aligned}
e^{0^{\prime}} & =d t, \\
e^{1^{\prime}} & =\kappa a(t) d(\kappa \psi), \\
e^{2^{\prime}} & =\kappa a(t) \sin (\kappa \psi) d \theta, \\
e^{3^{\prime}} & =\kappa a(t) \sin (\kappa \psi) \sin \theta d \phi .
\end{aligned}
$$

However, this choice turn out to be incompatible with the field equations coming from the actions (13) and (14), because the vierbein (17) does not correctly parallelize the spacetime. The symptom that the choice (17) will not work is the form acquired by the Weitzenböck invariant in such case, which turn out to be

$$
T=2\left[(\kappa a)^{-2} \cot ^{2}(\kappa \psi)-3 H^{2}\right] .
$$

This form of $T$ would be unable of giving a proper reduced Lagrangian for the dynamics of the scale factor $a(t)$, as a consequence of its dependence on $\psi$. This $\psi$-dependent Weitzenböck invariant is not consistent with the isotropy and homogeneity of the FRW cosmological models.

We shall here sumarise the technique in order to obtain the parallelized frames. For details, we refer the reader to Ref. 27.

The procedure starts taking the naive frame of Eq. (17) and performing on it a suitable local Lorentz transformation. This transformation will locally twist the diagonal frame (17) to convert it in a global well defined parallel field. For the closed Universe $(\kappa=1)$, the local Lorentz transformation consist in a local rotation of the spatial tetrad $e^{1^{\prime}}, e^{2^{\prime}}, e^{3^{\prime}}$, with Euler angles $\psi, \theta, \phi$; thus both frames are related via the Euler matrix

$$
e^{a}=\mathcal{R}_{a^{\prime}}^{a} e^{a^{\prime}}
$$

where

$$
\mathcal{R}=\left(\begin{array}{cccc}
1 & 0 & 0 & 0 \\
0 & 1 & 0 & 0 \\
0 & 0 & \cos \phi & \sin \phi \\
0 & 0 & -\sin \phi & \cos \phi
\end{array}\right)\left(\begin{array}{cccc}
1 & 0 & 0 & 0 \\
0 & \cos \theta & \sin \theta & 0 \\
0 & -\sin \theta & \cos \theta & 0 \\
0 & 0 & 0 & 1
\end{array}\right)\left(\begin{array}{cccc}
1 & 0 & 0 & 0 \\
0 & 1 & 0 & 0 \\
0 & 0 & \cos \psi & \sin \psi \\
0 & 0 & -\sin \psi & \cos \psi
\end{array}\right)
$$


As usual, the angular coordinates range in the intervals $0 \leq \phi<2 \pi, 0 \leq \theta \leq \pi$ and $0 \leq \psi \leq \pi$. The aspect of the so locally rotated frame is now

$$
\begin{aligned}
& \stackrel{\circ}{E}^{1}=-c(\theta) d \psi+s(\psi) s(\theta)(c(\psi) d \theta-s(\psi) s(\theta) d \phi) \\
& \stackrel{\circ}{E}^{2}=s(\theta) c(\phi) d \psi-s(\psi)[(s(\psi) s(\phi)-c(\psi) c(\theta) c(\phi)) d \theta+ \\
& +(c(\psi) s(\phi)+s(\psi) c(\theta) c(\phi)) s(\theta) d \phi] \\
& \stackrel{\circ}{E}^{3}=-s(\theta) s(\phi) d \psi-s(\psi)[(s(\psi) c(\phi)+c(\psi) c(\theta) s(\phi)) d \theta+ \\
& +(c(\psi) c(\phi)-s(\psi) c(\theta) s(\phi)) s(\theta) d \phi],
\end{aligned}
$$

where, of course, $s \equiv \sin$ and $c \equiv \cos$.

A similar procedure is applicable to the open FRW Universe; take the same rotation (19) starting from the spatial part of the naive vierbein (17) with $\kappa=i$, but now replace the Euler angle $\psi$ by $i \psi$. The transformed fields read (here we put sh $\equiv \sinh$ and $c h \equiv \cosh )$

$$
\begin{aligned}
\breve{E}^{1}=c(\theta) d \psi+\operatorname{sh}(\psi) s(\theta)(-\operatorname{ch}(\psi) d \theta+i \operatorname{sh}(\psi) s(\theta) d \phi) \\
\breve{E}^{2}=-s(\theta) c(\phi) d \psi+\operatorname{sh}(\psi)[(i \operatorname{sh}(\psi) s(\phi)-\operatorname{ch}(\psi) c(\theta) c(\phi)) d \theta+ \\
+(\operatorname{ch}(\psi) s(\phi)+i \operatorname{sh}(\psi) c(\theta) c(\phi)) s(\theta) d \phi] \\
\breve{E}^{3}=s(\theta) s(\phi) d \psi+\operatorname{sh}(\psi)[(i \operatorname{sh}(\psi) c(\phi)+\operatorname{ch}(\psi) c(\theta) s(\phi)) d \theta+ \\
+(\operatorname{ch}(\psi) c(\phi)-i \operatorname{sh}(\psi) c(\theta) s(\phi)) s(\theta) d \phi] .
\end{aligned}
$$

Then, as the frame field of 1 -forms we take

$$
e^{0}=d t ; e^{1}=a(t) E^{1} ; e^{2}=a(t) E^{2} ; e^{3}=a(t) E^{3},
$$

where the $E^{i}$ 's allude to the dreibein $\stackrel{\circ}{ }^{i}$ and $\breve{E}^{i}, i=1,2,3$, of Eqs. (20) and (21) respectively. The fact that the naive vierbein (17) is connected with (22) by a local rotation guarantees that the latter still describes the spatially curved FRW metric given in Eq. (16).

The Weitzenböck invariant associated to the vierbein (22) is

$$
T=6\left( \pm a^{-2}-H^{2}\right),
$$

where the positive sign corresponds to the closed case and the negative to the open one, and $H=\dot{a} / a$ is the Hubble rate. ${ }^{\text {a }}$ The absence of angular vestiges in (23) foretell that the vierbein (22) will be adequate to solve the dynamical equations. Let us prove this statement first for the $f(T)$ schemes represented by the action $(13)$.

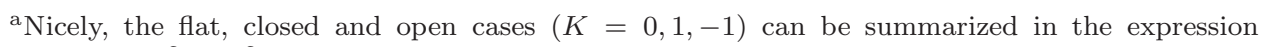
$T=6\left(K a^{-2}-H^{2}\right)$. See Ref. 3 for the flat case. 
For this purpose, we must replace the vierbein (22) in the field equations coming from the variation of the action (13) with respect to the components of the vierbein. When varying with respect to the temporal component we obtain the modified version of Friedmann equation, which turns out to be

$$
12 H^{2} f^{\prime}(T)+f(T)=16 \pi G \rho .
$$

The non trivial equations for the spatial sector are equal to

$$
4\left( \pm a^{-2}+\dot{H}\right)\left(12 H^{2} f^{\prime \prime}(T)+f^{\prime}(T)\right)-f(T)-4 f^{\prime}(T)\left(2 \dot{H}+3 H^{2}\right)=16 \pi G p .
$$

In the right hand side of the equations appear the energy density $\rho$ and the pressure $p$ of the fluid that fills the spacetime in a homogeneous and isotropic way, i.e., the energy-momentum tensor reads $T_{\mu}^{\nu}=\operatorname{diag}(\rho,-p,-p,-p)$ in the comoving frame. Note that Eq. (24) is of first order in time derivatives of the scale factor, irrespective of the function $f$. Equations (24) and (25) are two differential equations for just one unknown function $a(t)$; so, they cannot be independent. The way to see that this is indeed the case, is to take the time derivative of (24) and combine it with the conservation equation,

$$
\dot{\rho}=-3 H(\rho+p),
$$

to obtain Eq. (25). Conversely, if the system (24) and (25) is consistent, then the conservation of energy in the matter sector is given automatically and Eq. (26) holds. A further characterization of the scale factor dynamics depends on the particular choice of the function $f(T)$, but the general considerations about the correctness of the frames $(22)$ are valid for all $f$ 's.

The frames (22) are also valid for other theories with absolute parallelism, because they reflect the procedure of parallelization performed in the FRW manifold. We shall use them now in order to study the dynamics of the curved FRW models in four dimensional Born-Infeld gravity. For simplicity, we will work in the subspace of the parameter space $(A, B)$ defined by the condition $2 A+B=0$ (see Eq. (15)).

The dynamics of the scale factor is encoded in the modified Friedmann equation, which emerges from the replacement of the ansatz (22) in the motion equation coming from varying the action (14) with $n=4$ respect the temporal component of the tetrad $e^{0}$, and it looks

$$
\frac{\left|1 \pm \frac{1}{\lambda a^{2}}\right|^{3 / 2}}{\sqrt{1-\frac{12 H^{2}}{\lambda}}}-1=\frac{16 \pi G}{\lambda} \rho .
$$

In the last equation, the signs \pm refer again to the close and open cases respectively, and the bars mean absolute value. Again, the equation coming from the spatial sector is just the time derivative of (27) combined with the conservation equation (26), so we can ignore it in the analysis.

An exact solution of the motion equation (27) is beyond our present scope. However, the main physical features can be derived if one thinks about Eq. (27) as 
an energy conservation equation of the type

$$
\dot{y}^{2}+V(y)=0 \quad y=\frac{a}{a_{0}} .
$$

The potential $V(y)$ can be found easily, and it can be written as

$$
V(y)=-\frac{\lambda}{12} y^{2}\left[1-\frac{\left(1 \pm \gamma y^{-2}\right)^{3}}{\left(1+\beta y^{-3(1+\omega)}\right)^{2}}\right],
$$

where we have defined the two constants $\gamma=1 / \lambda a_{0}^{2}$ and $\beta=16 \pi G \rho_{0} / \lambda$. Additionally, the two constants $a_{0}$ and $\rho_{0}$ indicate present date values of the involved quantities, and $\omega$ is the barotropic index of the perfect fluid with state equation $p=\omega \rho$.

In Fig. 1 we can see the potentials (29) for a radiation-dominated Universe (i.e., $\omega=1 / 3)$, with $a_{0}=16 \pi G \rho_{0}=1$, so $\beta=\gamma=\lambda^{-1}$. The curves in black refers to the closed case and the ones in grey to the open case. The behavior of GR is depicted in dashed lines for both cases. While the GR potentials become divergent as $y \rightarrow 0$, we see that the BI dynamics behave drastically different in that (high energy) limit. As the scale factor tends to zero, the BI potentials go to zero with null derivative. In Fig. 1, we display the curves for three nominal values of the BI parameter, $\lambda=10^{3}$, $\lambda=10^{4}$ and $\lambda=10^{5}$. Note that the energy level in Eq. (28) is null, so the closed Universe possesses a turning point in the low energy regime, where the potential is null. The non divergence of the BI potentials in the regime when $y \rightarrow 0$ denotes a very nice feature of the theory. Actually, in that limit we have

$$
V(y)=-\frac{\lambda}{12} y^{2}+\mathcal{O}\left(y^{4}\right)
$$

so the Eq. (28) has, in the same limit, the solution

$$
a(t) \propto \operatorname{Exp}\left(H_{\max } t\right), \quad H_{\max }=\sqrt{\frac{\lambda}{12}} .
$$

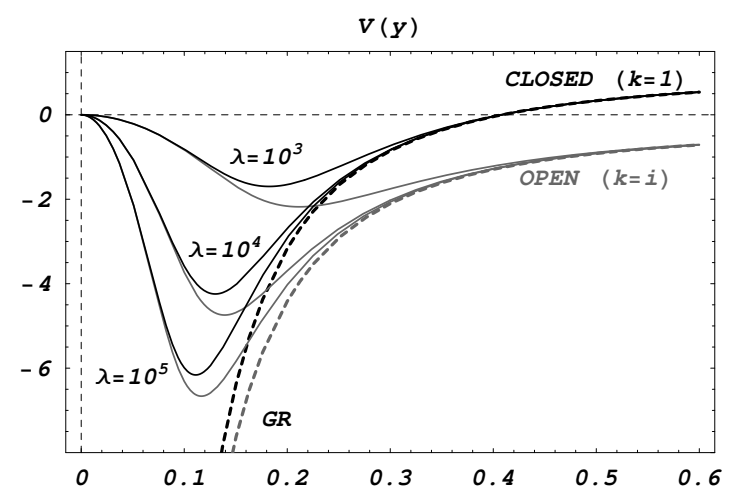

Fig. 1. Potentials for open (grey) and closed (black) radiation-dominated FRW Universes in Born-Infeld gravity, as emerge from Eq. (29), for several values of the BI parameter $\lambda$. The dashed curves correspond to the low energy limit of the theory, i.e., to GR. 
Then, in Born-Infeld cosmology we obtain that the early Universe is characterized by an inflationary stage of geometrical nature. The exponential behavior of the scale factor is driven by the BI parameter through the maximum Hubble rate defined according to $H_{\max }=(\lambda / 12)^{1 / 2}$. The existence of this early inflationary stage does not require the presence of the inflaton, and it seems to be an intrinsic feature of the BI regularization procedure. In fact the spacetime so obtained is regular and there is not Big Bang singularity in it, because the scale factor tends to zero when the proper time $t \rightarrow-\infty$. ${ }^{\mathrm{b}}$

\section{Final Remarks}

In this article we have discussed certain conceptual features related with the lack of local Lorentz invariance in gravitational theories with absolute parallelism and illustrated its consequences by means of two examples. In every gravitational theory possessing absolute parallelism, the spacetime structure is materialized in the coframe field $\left\{e^{a}\right\}$ which defines an orthonormal basis in the cotangent space $T_{p}^{*} M$ of the manifold $M$ at each point $p \in M$. As an example we analyzed the dynamics of FRW models in the so called $f(T)$ theories, which are enjoying a growing popularity in our days. When $f(T)=T$, i.e., when one considers general relativity in Weitzenböck spacetime, the basis $\left\{e^{a}\right\}$ at two different points of the manifold are completely uncorrelated, and it is not possible to define a global smooth field of bases unambiguously. This is so because the theory is invariant under the local Lorentz group acting on the coframes $\left\{e^{a}\right\}$. In turn, when $f(T) \neq T$, local Lorentz rotations and boosts are not symmetries of the theory anymore. Because of this lack of local Lorentz symmetry, the theory picks up a preferential global reference frame constituted by the coframe field $\left\{e^{a}\right\}$ that solves the field equations. In such case, the bases at two different points become strongly correlated in order to realize the parallelization of the manifold. Nevertheless, the appearance of a preferred reference frame is a property coming from the symmetries of the spacetime, and it is not ruled by the specific form of the function $f(T)$. For instance, when one is dealing with FRW cosmological spacetimes, the field of frames that will lead to consistent field equations will be given by (22), whatever the function $f(T)$ be.

Additionally, the fields (22) are also valid in more general theories with absolute parallelism which are not related with the $f(T)$ schemes, for instance, Born-Infeld gravity (14). In this framework, we have seen that the behavior of the early Universe is characterized by an inflationary stage of infinite duration and geometrical character. The exponential form of the scale factor is ruled by the BI parameter $\lambda$ and it does not rely on the existence of the inflaton. Finally, and due to the very nature of the Born-Infeld action, the spacetime results free of the Big Bang singularity.

b Though not shown here, all this properties are shared by the model with flat spatial section. 


\section{Acknowledgments}

F. Fiorini wants to thank the organizers of the 8th Alexander Friedmann International Seminar for their hospitality at Rio de Janeiro. This research was supported by CONICET and Universidad de Buenos Aires.

\section{References}

1. F. W. Hehl, P. von der Heyde, G. D. Kerlick and J. M. Nester, Rev. Mod. Phys. 48, 393 (1976).

2. F. W. Hehl, J. D. McCrea, E. W. Mielke and Y. Ne'eman, Phys. Rep. 258, 1 (1995).

3. R. Ferraro and F. Fiorini, Phys. Rev. D 75, 084031 (2007).

4. R. Ferraro and F. Fiorini, Phys. Rev. D 78, 124019 (2008).

5. F. Fiorini and R. Ferraro, Int. J. Mod. Phys. A 24, 1686 (2009).

6. G. Bengochea and R. Ferraro, Phys. Rev. D 79, 124019 (2009).

7. E. V. Linder, Phys. Rev. D 81, 127301 (2010); 82, 109902 (2010).

8. P. Wu and H. Yu, Phys. Lett. B 692, 176 (2010).

9. P. Wu and H. Yu, Phys. Lett. B 693, 415 (2010).

10. P. Wu and H. Yu, Eur. Phys. J. C 71, 1552 (2011).

11. K. K. Kerzhavov, S. R. Myrzakul, I. I. Kulnazarov and R. Myrzakulov, Accelerating cosmology in $\mathrm{F}(\mathrm{T})$ gravity with scalar field, arXiv:1006.3879v1.

12. R.-J. Yang, New types of $\mathrm{f}(\mathrm{T})$ gravities, arXiv:1007.3571v1.

13. R.-J. Yang, Europhys. Lett. 93, 60001 (2011).

14. K. Karami and A. Abdomaleki, Original and entropy-corrected versions of the holographic and new agegraphic $\mathrm{f}(\mathrm{T})$-gravity models, arXiv:1009.2459v1.

15. K. Karami and A. Abdomaleki, Reconstructing $\mathrm{f}(\mathrm{T})$-gravity from the polytropic and different Chaplygin gas dark energy models, arXiv:1009.3587v1.

16. J. B. Dent, S. Dutta and E. Saridakis, Phys. Rev. D 83, 023508 (2011).

17. J. B. Dent, S. Dutta and E. Saridakis, JCAP 1101, 009 (2011).

18. Y-F. Cai, S-H. Chen, J. B. Dent, S. Dutta and E. Saridakis, Matter bounce cosmology with the $\mathrm{f}(\mathrm{T})$ gravity, arXiv:1104.4349v1.

19. G. Bengochea, Phys. Lett. B 695, 405 (2011).

20. K. Bamba, C.-Q. Geng, C.-C. Lee, L.-W. Luo, JCAP 1101, 021 (2011).

21. P. B. Khatua, S. Chakraborty and U. Debnath, Dilaton dark energy model in $f(R)$, $\mathrm{f}(\mathrm{T})$ and Horava-Lifshitz gravities, arXiv:1105.3393v1.

22. R. Zheng and Q.-G. Huang, JCAP 1103, 002 (2011).

23. B. Li, T. P. Sotiriou and J. D. Barrow, Phys. Rev. D 83, 064035 (2011).

24. T. P. Sotiriou, B. Li and J. D. Barrow, Phys. Rev. D 83, 104030 (2011).

25. B. Li, T. P. Sotiriou and J. D. Barrow, Phys. Rev. D 83, 104017 (2011).

26. T. Wang, Static solutions with spherical symmetry in $f(T)$ theories, arXiv:1102.4410v1.

27. R. Ferraro and F. Fiorini, Non trivial frames for $\mathrm{f}(\mathrm{T})$ theories of gravity and beyond, arXiv:1103.082v1].

28. R. Ferraro and F. Fiorini, Phys. Lett. B 692, 206 (2010).

29. K. Hayashi and T. Shirafuji, Phys. Rev. D 19, 3524 (1979).

30. J.W. Maluf and J.F. da Rocha-Neto, Phys. Rev. D 64, 084014 (2001). 